\title{
Design of TMS320F28335 Based Simulation System of Fire-Fighting Equipments Manual-Control-Pad
}

\author{
Hao $\mathrm{Fu}^{1, \mathrm{a}^{*}}$, Yixin $\mathrm{Su}^{2, \mathrm{~b}}$ \\ School of Automation, Wuhan University of Technology, Wuhan, Hubei, 430000, China \\ a375258231@qq.com, b937340186@qq.com
}

Keywords: TMS320F28335, fire-fighting equipments, manual-control-pad, W5300

Abstract: In order to train the workers of power stations, a simulation system of the fire-fighting equipments manual-control-pad is designed with a high-performance 32-bit CPU TMS320F28335 and an Ethernet chip W5300. The simulation system will start or stop 8 virtual fire fighting equipments by operating the manual- control-pad, so as to simulate the actual fire alarm system. In this system, start signal or stop signal is sent to the upper computer through the Ethernet to start or stop the virtual fire-fighting equipments in it. This article elaborates the overall structure and working principles of the simulation system and puts some emphasis on the hardware principles as well as the software design strategies of the W5300 Ethernet interface. It also explains the flow chat of the software program. Practice indicates that this simulation system is able to work steadily and reliably.

\section{Introduction}

Potential risks exist in places like power stations whose internal structure is complex, it's high time that we insured its safe and reliable operation. Once fire occurs in power station, the consequence it might bring is serious. Therefore, strict requirements for the operators' field operation are put forward and there is no room for error. The operators at post should receive high-level education and training, have superior safety consciousness and conditioned reflex to the fire alarm. For these reasons, a fire alarm simulation training system seems to be of great significance [1].

The fire-fighting equipment manual-control-pad simulation system is a main part of the fire alarm simulation training system. When the operators receive fire alarm signals or trouble signals, they are able to start or stop 8 fire fighting equipments (fume extractors, fire pumps, etc.) quickly and abate fire or troubles.Fire-fighting equipment manual-control-pad simulation system is consistent with the actual control pad in aspects of operation interface and function which makes it convenient for instructors to train the operator' skills and inculcate enough safety awareness in them. Apparently, this simulation system has practical implications.

\section{System Structure and Main Function}

\section{A. System Structure.}

The fire-fighting equipments manual-control-pad simulation system is mainly consisted of the following parts: TMS320F28335, the W5300 Ethernet interface, the LED module, the keyboard module and the upper computer. Its system structure figure is shown as Fig.1.

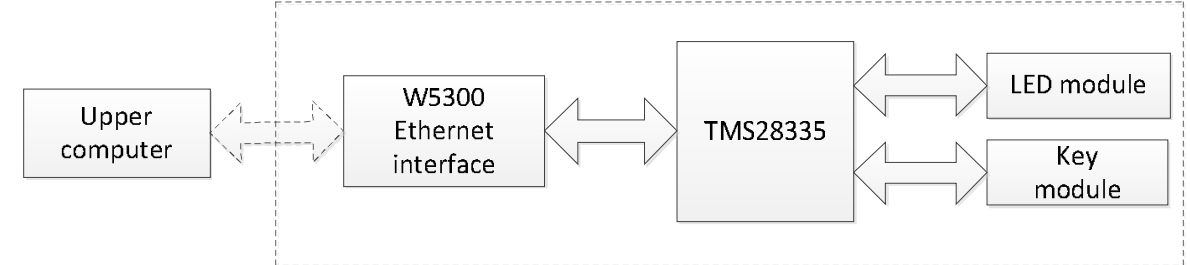

Fig.1 System structure figure of the fire-fighting equipments manual-control-pad simulation system.

A high-performance 32-bit CPU TMS320F28335 is selected as the main control chip. The CPU, whose clock frequency reaches up to $150 \mathrm{MHz}$, has many advantages such as fast processing rate, high precision, high performance, high peripheral integration, low cost, low power, large memory and fast 
response to interrupt. TMS320F28335 and W5300 share the sequential waveforms of CS signals, R/W signals, data bus and address bus, so it's achievable for TMS320F28335 to connect and communicate with the position machine through the W5300 Ethernet interface. When the fire alarm signal occurs and the time is limited, TMS320F28335's advantage of fast response to interrupt stands out [2].

\section{B. Main Function.}

When the operator receives fire alarm signals or trouble signals of a specific spot, he is supposed to start the corresponding fire fighting equipments or solve the trouble through the manual control pad. The manual control pad is consisted of 16 keys and 24 LEDs which are divided into 8 groups, it's to say, every 2 keys and 3 LEDs are in a peer group. 8 groups are used to control 8 fire-fighting equipments. The manual control pad is connected to the upper computer by the cable. When the operator press the start key of a certain group, the corresponding start LED and running LED are turned on, and dynamic diagrams of the fire fighting equipments at the corresponding spot are shown in the upper computer's interface. If the upper computer sends trouble signals to the manual control pad, the corresponding trouble LED is turned on. In this case, the operator should find and eliminate the troubles in time.

\section{Design of System Hardware}

\section{A. Design of W5300 Ethernet Interface.}

W5300 is a chip with integration of 10/100M Ethernet Controller, MAC and TCP/IP protocol stack. It's convenient, steady, and is widely used in the high-performance, low cost embedded fields. W5300 is easy to be connected with the CPU by the Bus Interface in direct access mode or indirect access mode. The load of CPU decreases for the reason of W5300's ability to process communication protocol in it, therefore, the performance of CPU improves significantly. The schematic diagram of the hardware interface betweenW5300 and TMS320F28335 is shown in Fig2.

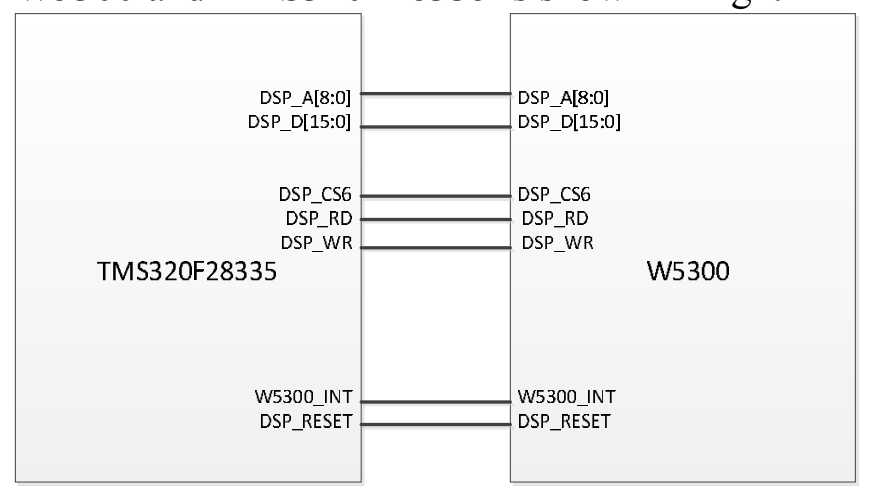

Fig. 2 The schematic diagram of the hardware interface betweenW5300 and TMS320F28335.

In the design, W5300's TEST_MODE [3:0] pins and OP_MODE [2:0] pins are grounded to set it as the internal PHY mode (normal running mode). Connect its address bus, data bus, the CS pin, the $\mathrm{R} / \mathrm{W}$ pin, the interrupt pin and the reset pin with the corresponding pins of TMS320F28335. Connect the differential input signals RXIP, RXIN and the differential output signals TXOP, TXON with the HR911105A cable interface. With the addition of power and ground, the hardware interface of W5300 is available [3].

\section{B. Design of Keys and LEDs Modules}

24 LEDs are divided into three groups. With the addition of current-limiting resistances, 8 LEDs with common anode in each group are connected to the GIOPs of TMS320F28335 through a bi-directional tri-state data buffer. $74 \mathrm{HC} 245$ is an eight-channel tri-state data buffer with controlled direction. Its main function is to realize the two-way asynchronous communication of the data bus. Data buffer is often applied to protect the fragile main control chip.16 keys are connected between the power and ground through current-limiting resistances. 


\section{Design of System Software}

\section{A. Design of Main Program}

System software includes W5300 Ethernet Interface program and key-LED interrupt program. The main program achieves the following function: the initialization of variables, DSP's peripheral and W5300, etc. The initialization of variables mainly defines some flag bits to make it convenient to execute, read and modify the programs. The initialization of DSP's peripheral open or shield some function of DSP to ensure the execution of the following program and the initialization of W5300 is the basis of Ethernet connection. The flow chart of the main program is shown in Fig.3 (a).

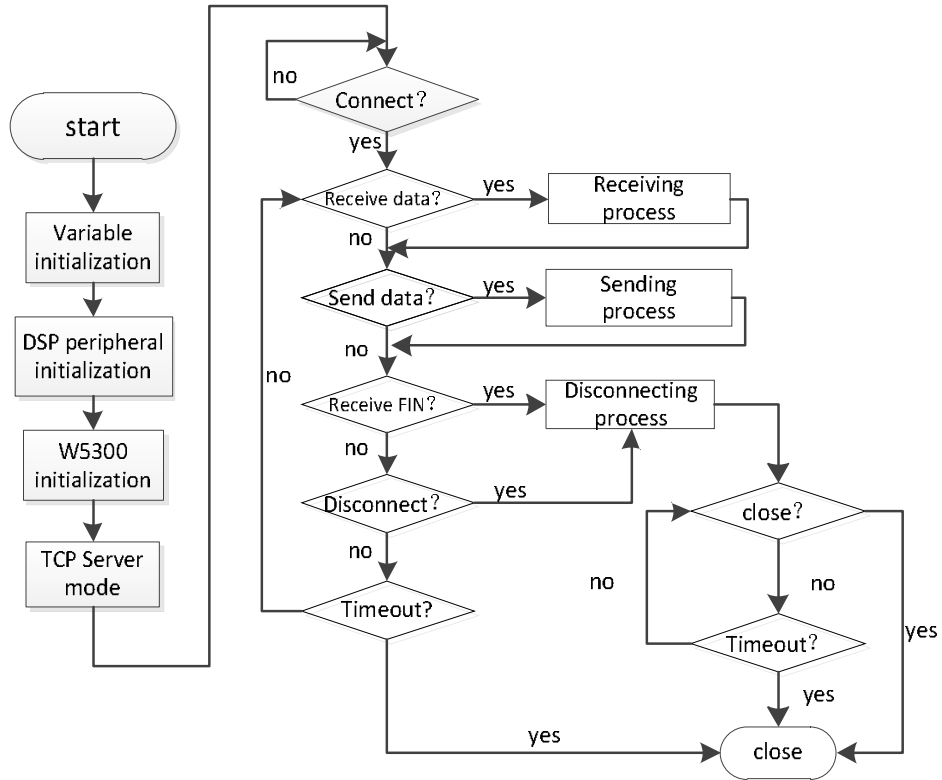

(a)

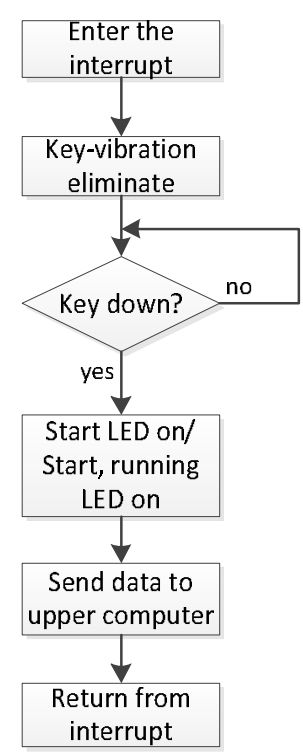

(b)

Fig.3 Flow chart of the main program and the key-LED interrupt program

\section{B. Design of W5300 Ethernet Interface Program.}

The initialization of W5300 includes three steps: the host settings, network information settings and the allocation of TX/RX storage. During initialization, W5300 is set as direct address mode with 16-bit bus width. The data bus exchange and FIFO exchange are prohibited. Enable the interrupt of socket0 and disable the rest. Then we set the gateway address of local CPU as"192.168.0.254", set the subnet mask as"255.255.255.0", set the IP address as"192.168.0.1". When data bags are failed to be sent, W5300 will repeat the sending process at a time interval of $200 \mathrm{~ms}$, for 8 times. Finally, TX/RX storage is allocated and the size of TX/RX of socket 0 is defined. After the steps above-mentioned, the initialization is completed.

After the initialization, W5300 is supposed to open sockets and exchange data via TCP. TCP is a kind of communication protocol. In TCP mode, we should firstly establish the connection to the end port according to the IP and socket number. In this way, date could be sent or received via the socket. When we establish a socket connection, "TCP server mode" or "TCP client mode" can be chosen. "TCP server" awaits connection request from the end port passively while "TCP client" asks for connection actively. In this design, the manual control pad is set as "TCP server mode" and awaits the connection request from the upper computer.

As is shown in Fig.3, after setting W5300 as "TCP server mode", several judgments are followed: connect or not, receive data or not, send data or not, receive FIN (end signal) or not, disconnect or not, timeout or not. If there is data to be sent or received, the process of sending or receiving data will be executed. The connection will be maintained until FIN is received or the socket is disconnected [4].

\section{Design of Key-LED Interrupt Program}

The key and LED program is designed with timer interrupt. When the program enters the interrupt, key-vibration is eliminated. Then judge whether the key is pressed or not. If the start key is pressed, the start LED will be on and start signal will be sent to the upper computer; if the stop key is pressed, the 
start LED and running LED will be off and stop signal will be sent to the upper computer. Finally, the program returns from interrupt. The flow chart of key-LED interrupt program is shown as Fig.3(b).

\section{The Experimental Result}

After setting the IP of the upper computer and connecting to the Ethernet with a cable, tests can be started. Firstly click the "connect" button in the upper computer, when "connect to server" appeared in the $\log$, connection is established. Under normal condition, if the start key of the fume extractor in "room one" is pressed, the start, running LEDs will be on and a dynamic graph of the fume extractor will be vividly shown in the upper computer, as is shown in Fig.4(a), Fig.5(a). Click the picture of fume extractor and a "red cross" appears, this indicates the trouble condition. This time when the start key is pressed, the red start LED and yellow trouble LED is on but the fume extractor fails to work, as is shown in Fig .4(b), Fig.5(b). The simulating control of the other equipments is analogous.

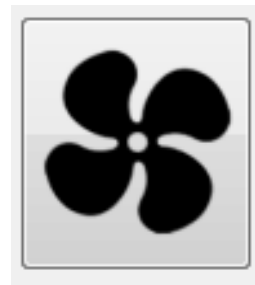

(a)

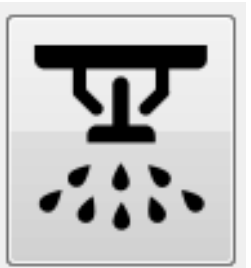

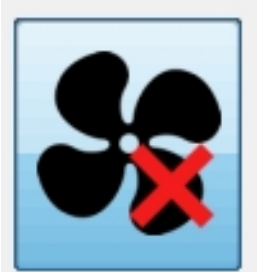

(b)

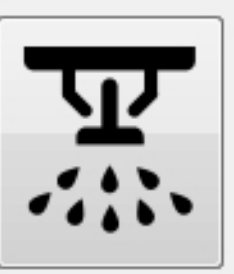

Fig.4 upper computer and manual control pad under normal condition

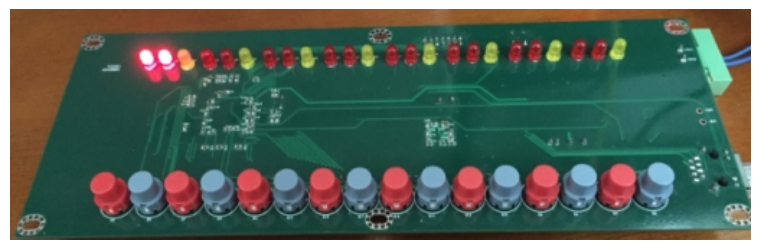

(a)

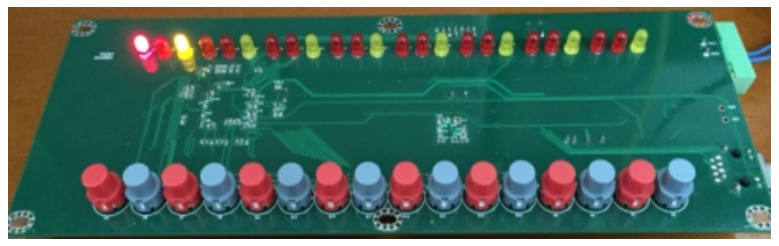

(b)

Fig.5 upper computer and manual control pad under trouble condition

\section{Conclusion}

The simulation system of the fire-fighting equipment manual-control-pad use TMS320F28335 as CPU. Its high-speed data processing ability ensures the timeliness of sending and receiving data. High-performance Ethernet chip W5300 with integration of TCP protocol decreases the load of CPU, so the performance of the CPU increases significantly. The result of the test indicates that the simulation system achieves the ideal function and works steadily. It is feasible to use it as the simulation training system in power stations.

\section{References}

[1] Pingsheng Xu. Simulator Training and Licensing Examination for Nuclear Power Station Operator [J].Nuclear Power Engineering, 2007, 28(4):108-111,121.

[2]Texas Instruments.TMS320F28335, TMS320F28334, TMS320-F28332 Data Sheet / http://www-s.ti.com/sc/techlit. 2007.

[3] Quanmei Ou. The design and realization of embedded Ethernet. Computer Knowledge and Technology, 2007(6):35-37

[4] WIZnet Device,Inc.High-performance internet connectivity solution-W5300[EB/OL].(2008).

http://www.wiznet.cokr. 